\title{
Body Representation Alterations in Patients with Unilateral Brain Damage
}

\author{
Simona Raimo ${ }^{1,2, *}$ (1) , Maddalena Boccia ${ }^{2,3}$, Antonella Di Vita ${ }^{2,3}$, Teresa Iona ${ }^{1}$, Maria Cropano ${ }^{4}$, Antonio Ammendolia ${ }^{1}$, \\ Roberto Colao $^{5}$, Valentina Angelillo ${ }^{6}$, Antonio Maiorino ${ }^{6}$, Cecilia Guariglia $^{2,3}$, Dario Grossi ${ }^{4}$ and Liana Palermo ${ }^{1,2, *}$ \\ ${ }^{1}$ Department of Medical and Surgical Sciences, Magna Graecia University, Viale Europa - Loc. Germaneto, 88100, Catanzaro, Italy \\ ${ }^{2}$ I.R.C.C.S. Santa Lucia Foundation, Via Ardeatina 306, 00179, Rome, Italy \\ ${ }^{3}$ Department of Psychology, "Sapienza" University of Rome, via dei Marsi 78, 00185, Rome, Italy \\ ${ }^{4}$ Department of Psychology, University of Campania "Luigi Vanvitelli", Viale Ellittico 31, 81100, Caserta, Italy \\ ${ }^{5}$ Centro Aqua Salus, Via Frischia 137-139, 88050, Sellia Marina (Catanzaro), Italy \\ ${ }^{6}$ Rehabilitation Clinic, "Villa delle Magnolie", Via Ciummiento 37, 81020 Castel Morrone (Caserta), Italy
}

(Received May 12, 2020; Final Revision January 16, 2021; Accepted January 26, 2021; First Published Online March 5, 2021)

\begin{abstract}
Objective: Systematic studies about the impact of unilateral brain damage on the different body representations (body schema, body structural representation, and body semantics) are still rare. Aim of this study was to evaluate body representation deficits in a relatively large sample of patients with unilateral brain damage and to investigate the impact of right or left brain damage on body representations (BRs), independently from deficits in other cognitive processes. Method: Sixty-four patients with unilateral stroke (22 with left brain damage, LBD; 31 with right brain damage without neglect, RBD-N; 11 with right brain damage with neglect, $\mathrm{RBD}+\mathrm{N})$ and 41 healthy individuals underwent a specific battery including BR as well as control tasks. Results: In more than a third of the sample, selective (37.5\%) and pure $(31 \%)$ deficits of BR were presented and equally distributed among the different BRs $(\sim 10 \%$ for each representation), with selective $(27.2 \%)$ and pure $(22.7 \%)$ body schema deficit mainly presented after left brain damage. As a group, patients with unilateral brain damage, independently of the side of lesion (LBD, RBD-N, RBD+N), had significantly worse performance on body structural representation with respect to healthy individuals, whereas LBD had numerically worse performance on body schema with respect to healthy individuals and RBD-N. No significant differences among groups were found on body semantics. Conclusion: BR deficits are not a rare consequence of unilateral brain damage and are independent of a more general cognitive dysfunction. Accordingly, the need for an accurate assessment and specific neuropsychological training in clinical settings is discussed.
\end{abstract}

Keywords: Body representation, Body schema, Body structural representation, Body semantics, Stroke, Body image

\section{INTRODUCTION}

Body representation (BR) is a complex process involving various sources of top-down and bottom-up information (e.g., visual, proprioceptive, interoceptive) that is continuously and constantly updated (de Vignemont, 2011; Karnath \& Baier, 2010; Palermo, Di Vita, Piccardi, Traballesi, \& Guariglia, 2014). It is not a homogeneous concept, and several theoretical models have been proposed (Sirigu, Grafman, Bressler, \& Sunderland, 1991; Berlucchi \&

*Correspondence and reprint requests to: S. Raimo, Ph.D., Department of Medical and Surgical Sciences, Magna Graecia University of Catanzaro, Catanzaro, Italy. E-mail: simonaraimo.sun@gmail.com or simona.raimo@ unicz.it and L. Palermo, Ph.D., Department of Medical and Surgical Sciences, Magna Graecia University of Catanzaro, Catanzaro, Italy. E-mail: liana.palermo@unicz.it
Aglioti, 2010; Corradi-Dell'Acqua \& Rumiati, 2007). The two main taxonomies are the dyadic taxonomy (Paillard, 1980), which distinguishes between body schema and body image, and the triadic taxonomy (Schwoebel \& Coslett, 2005), which maintains the proposed subdivision although it splits further the body image notion into two components, namely topological map of the body and body semantics. Neuropsychological evidence of multiple dissociations among different BRs supports the triadic taxonomy, suggesting that three different types of BR contribute to body knowledge, as it follows: (i) the body schema, which refers to the dynamic representation of body parts and their relative positions and contributes to the performance of movements by providing information about the dynamic position of each body part in relation to other parts (Schwoebel, Boronat, \& Coslett, 2002; Schwoebel \& Coslett, 2005); (ii) the body structural representation (also 
called the visuospatial body map), which refers to a topological map of the body and contains data (mainly visual) about the location of body parts, the borders of the body, and distance relations between body parts (Buxbaum \& Coslett, 2001; Schwoebel \& Coslett, 2005); (iii) the body semantics, which refers to a lexical-semantic representation of the body and contains the names of body parts and their functions, relations, and associations with objects (Coslett, Saffran, \& Schwoebel, 2002; Schwoebel \& Coslett, 2005). Deficit of BRs has been analyzed mainly in single case reports or small group studies (i.e., Buxbaum \& Coslett, 2001; Guariglia, Piccardi, Allegra, \& Traballesi, 2002; Laiacona, Allamano, Lorenzi, \& Capitani, 2006; Palermo et al., 2014; Di Vita et al., 2017), showing that the impairment of a specific BR would be associated with a specific neuropsychological deficit. For example, the disruption of the body schema could result in ideomotor apraxia (Buxbaum, Giovannetti, \& Libon, 2000), the disruption of the body structural representation could result in autotopoagnosia (Sirigu et al., 1991) or personal neglect (Palermo et al., 2014; Di Vita et al., 2017; Committeri, Piervincenzi, \& Pizzamiglio, 2018), and the disruption of body semantics could contribute to body-specific aphasia (Suzuki, Yamadori, \& Fuji, 1997). Systematic group studies on different BRs in patients with unilateral brain damage are still rare, and to the best of our knowledge, only two studies have been reported in literature (Razmus, 2017; Schwoebel \& Coslett, 2005). The seminal study by Schwoebel \& Coslett (2005) systematically investigated different BRs in a large sample $(n=70)$ of unilateral braindamaged patients, showing that at least one BR was affected in more than a half of patients with stroke (51\% of patients). Body semantics and structural BR deficits were mainly associated with lesions lateralized to the left hemisphere, in particular with left temporal lesions, while no clear lateralization was observed for body schema deficits, mainly associated with parietal and dorsolateral frontal lesions. The study by Razmus (2017), instead, was performed on a mixed sample of bilateral ( $70 \%$ of the sample) and unilateral brain-damaged patients, and deficits in at least one BR were found in 38 out of 47 patients ( $81 \%$ of patients). Overall these data suggest that BR deficits are widespread after stroke. However, in these two previous studies, representation deficits not directly related to body processing were not evaluated, leaving open the possibility that the reported BR deficits were not specific but due to more general cognitive deficits (e.g., deficit in language processing, in visuospatial and mental imagery skills). Indeed, Razmus (2017) found associations between the performance in the BR tasks and deficits in working memory, visuospatial deficit, and naming of objects as evaluated with standard neuropsychological tests. Therefore, the purpose of the current study is to extend these findings conducting a systematic exploration of the impact of unilateral brain damage on BRs (i.e., body schema, body structural representation, and body semantics), using a specific battery of BR tests and comparing the performance of patients with right or left unilateral brain damage with that of a sample of healthy controls. Moreover, since a number of cognitive processes, beyond body processing, are necessary to perform BR tasks, we also administered similar control tasks, with no bodyrelated stimuli, to disentangle the assessment of BRs from other cognitive functions.

Here we were interested in studying these three BRs and not to evaluate the clinical manifestations of BR alterations. In other words, an evaluation of how BR deficits can be related to several bodily disorders (e.g., autotopagnosia, body form agnosia, autoscopy, somatoparaphrenia, etc.; for a full list and description of these disorders, see de Vignemont, 2010), was out of the scope of the current study, and we refer the interested readers to previous works for specific studies on these disorders and clinical accounts (for studies on specific bodily disorders of neurological origins - such as autoscopy, somatoparaphrenia, anosognosia for hemiplegia, phantom limb, and personal neglect - see, e.g., Blanke \& Mohr, 2005; Vallar \& Ronchi, 2009; Antoniello et al., 2010; Gandola et al., 2012; Moro et al., 2016; Committeri et al., 2018; for studies on several body awareness disorders, see Herbet et al., 2019).

\section{METHODS}

\section{Participants}

Sixty-four patients with unilateral stroke and 41 healthy individuals (control participants, $\mathrm{C}$ : mean age $=58.39, \mathrm{SD}=7.03$; mean education $=12.49, \mathrm{SD}=4.05$ ) were enrolled in the study. Patients were divided into two main groups according to the side of lesion (22 with left brain damage, LBD; and 42 with right brain damage, RBD), and patients with RBD were, in turn, divided into two groups according to the presence of neglect (31 without neglect RBD-N; 11 right BD with neglect, $\mathrm{RBD}+\mathrm{N})$. Indeed $\mathrm{RBD}+\mathrm{N}$, compared with $\mathrm{RBD}-\mathrm{N}$, has shown to have distinctive clinical characteristics, such as deficit of awareness for visceral sensations (see Raimo et al., 2020), longer recovery, more functional disabilities (Paolucci et al., 2010), and distinct neural correlates (Committeri et al., 2007) that could affect BR performance. Age $\left(\chi_{(3)}^{2}=4.43 ; p=.218\right)$ and education $\left(\chi_{(3)}^{2}=6.92\right.$; $p=.074)$ did not differ among groups. Even though there was a trend toward significance, which suggested that $\mathrm{C}$ had higher educational level compared with LBD (mean education $=9.91, \mathrm{SD}=4.51 ; p=.058$ ) and $\mathrm{RBD}+\mathrm{N}$ (mean education $=9.73, \mathrm{SD}=2.61 ; p=.055)$. This trend was not observed for the patient groups (i.e., the LDB, $\mathrm{RBD}+\mathrm{N}$, and RBD-N showed the same level of education). Demographic information and neuropsychological data for patients are shown in Table 1.

Patients were recruited from a population of in-patients at the IRCCS Fondazione Santa Lucia (Rome), the Rehabilitation Clinic, "Villa delle Magnolie" (Caserta), and "Aqua Salus" Rehabilitation Center (Catanzaro). Exclusion criteria for the patients were: the presence of more than one cerebrovascular accident, neoplastic or traumatic aetiology, cognitive deterioration, histories of psychiatric illness or substance abuse, and severe language comprehension deficits 
Table 1. Patients' demographic and assessment data

\begin{tabular}{|c|c|c|c|c|c|c|c|}
\hline Participants & Group & Gender & Age (years) & Education (years) & Time post-onset & RMI & RCPM \\
\hline Pt 1 & LBD & M & 55 & 18 & SA & $5 / 15$ & $32 / 36$ \\
\hline Pt 2 & LBD & M & 72 & 5 & $\mathrm{Cr}$ & $6 / 15$ & $22 / 36$ \\
\hline Pt 3 & LBD & $\mathrm{F}$ & 70 & 5 & $\mathrm{Cr}$ & $11 / 15$ & $23 / 36$ \\
\hline Pt 4 & LBD & $\mathrm{F}$ & 48 & 8 & $\mathrm{Cr}$ & $5 / 15$ & $20 / 36$ \\
\hline Pt 5 & LBD & M & 66 & 8 & $\mathrm{Cr}$ & N.A. & N.A. \\
\hline Pt 6 & LBD & M & 61 & 8 & $\mathrm{Cr}$ & $7 / 15$ & $30 / 36$ \\
\hline Pt 7 & LBD & $\mathrm{F}$ & 59 & 5 & $\mathrm{Cr}$ & $13 / 15$ & $23 / 36$ \\
\hline Pt 8 & LBD & $\mathrm{F}$ & 68 & 3 & $\mathrm{Cr}$ & $12 / 15$ & N.A. \\
\hline Pt 9 & LBD & M & 71 & 8 & $\mathrm{Cr}$ & N.A. & $21 / 36$ \\
\hline Pt 10 & LBD & M & 55 & 18 & SA & N.A. & $33 / 36$ \\
\hline Pt 11 & LBD & M & 76 & 13 & SA & $4 / 15$ & $32 / 36$ \\
\hline Pt 12 & LBD & M & 62 & 10 & SA & $1 / 15$ & $26 / 36$ \\
\hline Pt 13 & LBD & M & 55 & 13 & SA & $1 / 15$ & $28 / 36$ \\
\hline Pt 14 & LBD & M & 51 & 13 & SA & $2 / 15$ & $20 / 36$ \\
\hline Pt 15 & LBD & M & 44 & 18 & SA & $3 / 15$ & $23 / 36$ \\
\hline Pt 16 & LBD & M & 52 & 11 & SA & $15 / 15$ & $29 / 36$ \\
\hline Pt 17 & LBD & M & 61 & 6 & SA & $7 / 15$ & $23 / 36$ \\
\hline Pt 18 & LBD & $\mathrm{F}$ & 60 & 16 & SA & $1 / 15$ & $21 / 36$ \\
\hline Pt 19 & LBD & $\mathrm{F}$ & 64 & 8 & SA & $1 / 15$ & $26 / 36$ \\
\hline Pt 20 & LBD & M & 75 & 8 & SA & $8 / 15$ & $24 / 36$ \\
\hline Pt 21 & LBD & M & 68 & 8 & SA & $7 / 15$ & $26 / 36$ \\
\hline Pt 22 & LBD & $\mathrm{F}$ & 57 & 8 & SA & $8 / 15$ & $25 / 36$ \\
\hline Pt 23 & RBD-N & M & 56 & 13 & SA & $1 / 15$ & $34 / 36$ \\
\hline Pt 24 & RBD-N & M & 78 & 8 & SA & $3 / 15$ & $21 / 36$ \\
\hline Pt 25 & RBD-N & M & 49 & 13 & SA & $8 / 15$ & $33 / 36$ \\
\hline Pt 26 & RBD-N & $\mathrm{F}$ & 51 & 18 & SA & N.A. & $33 / 36$ \\
\hline Pt 27 & RBD-N & M & 50 & 12 & SA & $4 / 15$ & $21 / 36$ \\
\hline Pt 28 & RBD-N & M & 48 & 13 & SA & $4 / 15$ & $30 / 36$ \\
\hline Pt 29 & RBD-N & $\mathrm{F}$ & 61 & 8 & SA & $1 / 15$ & $27 / 36$ \\
\hline Pt 30 & RBD-N & M & 68 & 18 & SA & $0 / 15$ & $29 / 36$ \\
\hline Pt 31 & RBD-N & M & 73 & 11 & SA & N.A. & N.A. \\
\hline Pt 32 & RBD-N & M & 66 & 13 & SA & N.A. & $30 / 36$ \\
\hline Pt 33 & RBD-N & M & 54 & 13 & SA & N.A. & $21 / 36$ \\
\hline Pt 34 & RBD-N & $\mathrm{F}$ & 44 & 13 & SA & $1 / 15$ & $19 / 36$ \\
\hline Pt 35 & RBD-N & $\mathrm{F}$ & 51 & 13 & SA & $8 / 15$ & $34 / 36$ \\
\hline Pt 36 & RBD-N & M & 76 & 13 & $\mathrm{Cr}$ & $5 / 15$ & N.A. \\
\hline Pt 37 & RBD-N & M & 65 & 18 & $\mathrm{Cr}$ & $11 / 15$ & $18 / 36$ \\
\hline Pt 38 & RBD-N & M & 69 & 16 & $\mathrm{Cr}$ & $4 / 15$ & $33 / 36$ \\
\hline Pt 39 & RBD-N & $\mathrm{F}$ & 73 & 10 & $\mathrm{Cr}$ & N.A. & $24 / 36$ \\
\hline Pt 40 & RBD-N & $\mathrm{F}$ & 42 & 13 & SA & N.A. & $25 / 36$ \\
\hline Pt 41 & RBD-N & M & 76 & 5 & SA & $0 / 15$ & $20 / 36$ \\
\hline Pt 42 & RBD-N & M & 72 & 14 & SA & $0 / 15$ & $23 / 36$ \\
\hline Pt 43 & RBD-N & M & 75 & 18 & SA & $0 / 15$ & $21 / 36$ \\
\hline Pt 44 & RBD-N & M & 53 & 8 & SA & $8 / 15$ & $29 / 36$ \\
\hline Pt 45 & RBD-N & M & 45 & 13 & SA & $15 / 15$ & $31 / 36$ \\
\hline Pt 46 & RBD-N & M & 58 & 8 & SA & $1 / 15$ & $26 / 36$ \\
\hline Pt 47 & RBD-N & $\mathrm{F}$ & 64 & 8 & SA & $0 / 15$ & $18 / 36$ \\
\hline Pt 48 & RBD-N & M & 62 & 8 & SA & $6 / 15$ & $22 / 36$ \\
\hline Pt 49 & RBD-N & $\mathrm{F}$ & 71 & 4 & SA & $1 / 15$ & $25 / 36$ \\
\hline Pt 50 & RBD-N & M & 73 & 12 & SA & $10 / 15$ & $26 / 36$ \\
\hline Pt 51 & RBD-N & M & 71 & 11 & SA & $2 / 15$ & N.A. \\
\hline Pt 52 & RBD-N & $\mathrm{F}$ & 66 & 8 & $\mathrm{Cr}$ & $7 / 15$ & $25 / 36$ \\
\hline Pt 53 & RBD-N & $\mathrm{F}$ & 48 & 8 & $\mathrm{Cr}$ & $14 / 15$ & $24 / 36$ \\
\hline Pt 54 & $\mathrm{RBD}+\mathrm{N}$ & $\mathrm{M}$ & 61 & 8 & SA & $1 / 15$ & $27 / 36$ \\
\hline Pt 55 & $\mathrm{RBD}+\mathrm{N}$ & $\mathrm{F}$ & 50 & 8 & SA & $0 / 15$ & $26 / 36$ \\
\hline Pt 56 & $\mathrm{RBD}+\mathrm{N}$ & $\mathrm{F}$ & 73 & 5 & SA & $6 / 15$ & N.A. \\
\hline Pt 57 & $\mathrm{RBD}+\mathrm{N}$ & M & 66 & 10 & SA & $3 / 15$ & $33 / 36$ \\
\hline
\end{tabular}


Table 1. (Continued)

\begin{tabular}{lccccccc}
\hline Participants & Group & Gender & Age (years) & Education (years) & Time post-onset & RMI & RCPM \\
\hline Pt 58 & RBD+N & M & 49 & 11 & SA & $1 / 15$ & $25 / 36$ \\
Pt 59 & RBD+N & F & 75 & 13 & SA & $2 / 15$ & N.A. \\
Pt 60 & RBD+N & M & 41 & 13 & SA & N.A. & $20 / 36$ \\
Pt 61 & RBD+N & M & 70 & 8 & Cr & $9 / 15$ & $22 / 36$ \\
Pt 62 & RBD+N & M & 63 & 13 & Cr & $6 / 15$ & N.A. \\
Pt 63 & RBD+N & M & 71 & 8 & Cr & $8 / 15$ & N.A. \\
Pt 64 & RBD+N & F & 73 & 10 & Cr & $7 / 15$ & $24 / 36$ \\
\hline \hline
\end{tabular}

Row scores are reported for the RCPM and after correction for age and education, all the scores were within the normal range. LBD, left-brain-damaged patients; RPN, right-brain-damaged patients without neglect; RBD+N, right-brain-damaged patients with neglect; SA, subacute phase (10-90 days from stroke); Cr, cronic phase ( $>90$ days from stroke); RMI, Rivermead Mobility Index; RCPM, Raven's Progressive Matrices; N.A., Not available.

that prevented them from understanding the experimental tasks. At the time of the testing, none of the patients or caregivers spontaneously reported symptoms compatible with somatoparaphrenia, kinaesthetic hallucinations, supernumerary phantom limbs, anosognosia, or limb personification. Hemiplegia/hemiparesis was present in $76 \%$ of patients. Controls were recruited from the local community, and they did not show signs of neurological and/or psychiatric diseases or general cognitive impairment and history of substance abuse. The study was carried out in accordance with the Declaration of Helsinki, and ethical approval was obtained from the Ethics Committees of the Fondazione Santa Lucia IRCCS (Rome), of the University of Campania "Luigi Vanvitelli" (Caserta), and Calabria Region Ethical Committee, Catanzaro, Italy. Informed written consent was obtained from all the participants.

\section{Neuropsychological Testing}

Two trained neuropsychologists conducted an initial screening interview to exclude patients who showed severe language comprehension impairments. The Raven's Coloured Progressive Matrices, (Raven, 1938; Spinnler \& Tognoni, 1987) was given to patients to exclude the presence of deficit in abstract reasoning. Extrapersonal and personal neglect (see the supplementary material 1 for more details) were evaluated with the Standard Battery for the Evaluation of Hemineglect (Pizzamiglio, Judica, Razzano, \& Zoccolotti, 1989; Guariglia, Palermo, Piccardi, Iaria, \& Incoccia, 2013) and the Use of Common Objects test (Zoccolotti et al., 1992). For a detailed description of the brain imaging data collection and results, see the supplementary material 2 .

\section{Body Representations Assessment}

Currently there is a lack of normative tests probing the different BRs. For this reason, BR tasks were carefully choose among experimental tasks that have proved to be very sensitive to detect BR deficit in several previous studies on adults with brain damage (Canzano, Piccardi, Bureca, \& Guariglia, 2011; Guariglia \& Antonucci, 1992; Schwoebel \& Coslett, 2005) and children with cerebral palsy (Fontes et al., 2017;
Di Vita et al., 2020). These tasks were also used in recent studies on the development of BRs in healthy children and young adults (Raimo et al., 2019) and on BR in older adults (Raimo et al., 2021).

\section{Assessment of Body Semantics}

Body semantics was evaluated using an Object-Body Part Association Task (adapted from Fontes, Moura, \& Haase, 2014; Schwoebel \& Coslett, 2005; Raimo et al., 2019). The task included 20 stimuli in which participants had to correctly associate an object (e.g., hat) to the relative part of the body, choosing between two options (e.g., head and foot). In particular, an object (target) in the middle of the touchscreen and different body parts (response items) on the left and right sides of the touchscreen were shown. The participant had to associate the body part with the target object, by tapping one of the two response items. The task included 20 stimuli. Individual scores range from 0 to 20 , with higher scores indicating better performance.

In the control task (i.e., a task involving the semantic processing of non-body-related stimuli), namely the ObjectRoom Association Task (Raimo et al., 2019), the participants had to correctly associate an object (e.g., armchair) to the relative room, choosing between two options (e.g., living room and bathroom). In particular, an object (target) in the middle of the touchscreen and two different rooms (response items) on the left and right sides of the touchscreen were shown. The participant had to associate the room with the target object, by tapping one of the two response items. The control task included 20 stimuli. Individual scores range from 0 to 20 with higher scores indicating better performance. Accuracy was recorded for both tasks, and the task presentation order was counterbalanced across participants. See Figure 1 for an example of the task items.

\section{Assessment of the Body Structural Representation}

The body structural representation was evaluated using a computerized version of the Frontal Body Evocation Task (FBE) of the BR test (Daurat-Hmeljiak, Stambak, \& Berges, 1978; Raimo et al., 2019) that included a frame, on which the head of a child was depicted, and nine body 
(A)
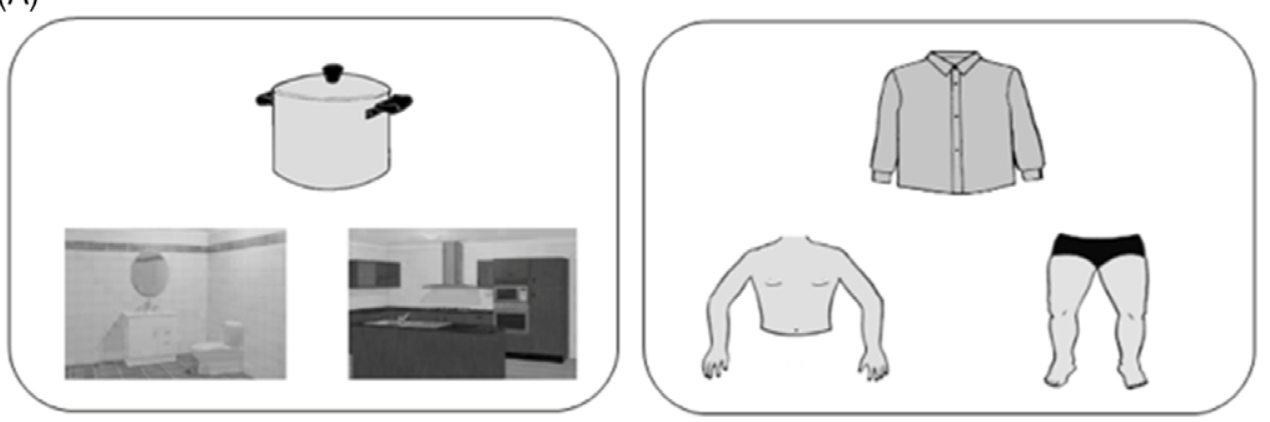

(B)
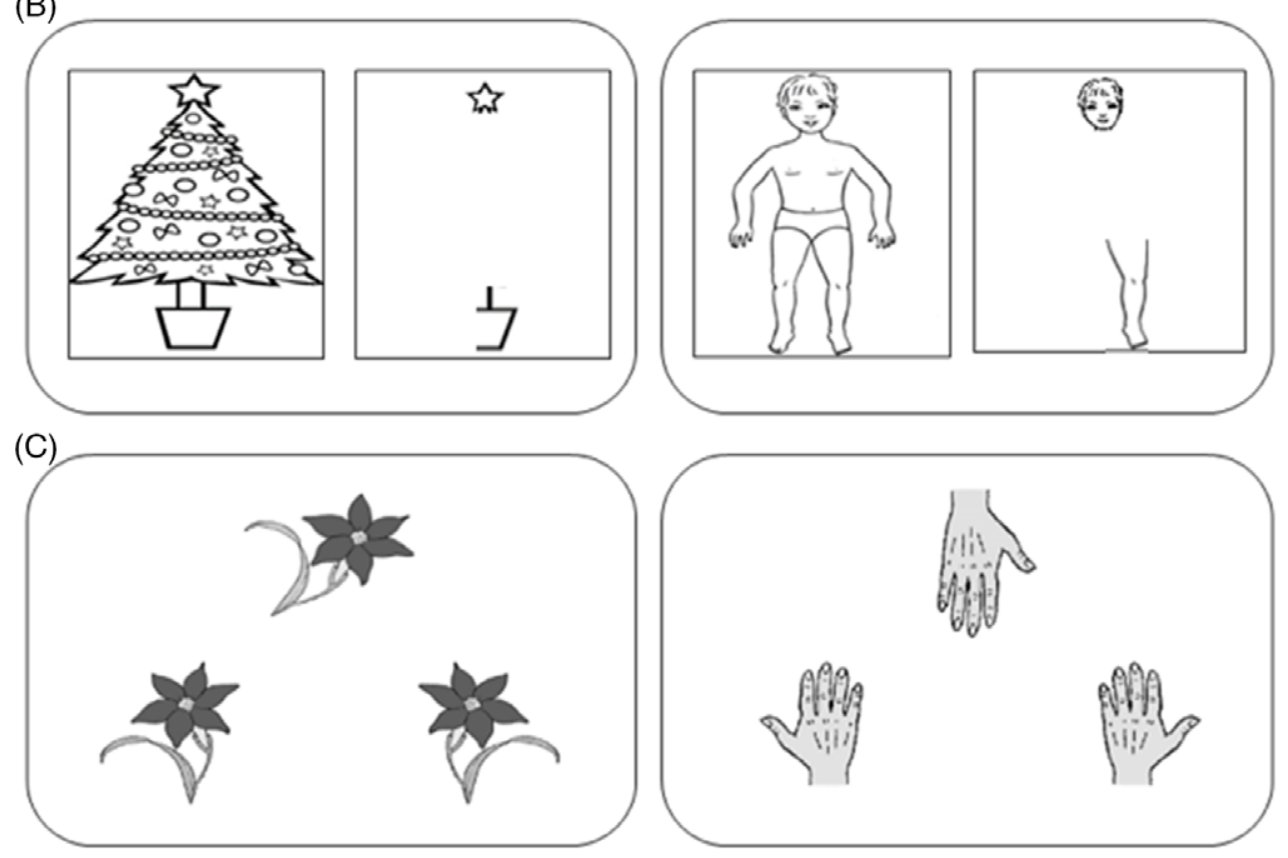

Fig. 1. Examples of items for the tasks involving body (right) and nonbody (left) processing. (A) An item of the task assessing the body semantics (Object-Body Part Association Task) is shown on the right panel, and an item of the control task (Object-Room Association Task) is shown on the left panel; (B) the task assessing the body structural representation (Frontal Body Evocation Task) is shown on the right panel and the control task (Christmas Three Task) on the left panel; (C) an item of the task assessing the body schema (Hand Laterality Task) is shown on the right panel, and an item of the control task (Object Laterality Task) is shown on the left panel.

parts (left and right legs, left and right hands, left and right arms, left and right parts of the chest and the neck). On a touch screen monitor, the participants were presented with one body part at a time, and their task was to place each part on the frame where only the head was depicted as reference part. In particular, a specific body part was shown on the right side of the frame and the participants had to place it at the correct position in the frame with respect to the reference of the head, by dragging it with their fingers. The position of the body part was recorded by the computer in terms of $\mathrm{mm}$ of distance from correct positioning; then, the body part disappeared and a new one was presented.

The control task (i.e., a task involving the visuospatial processing of non-body-related stimuli), namely the Christmas Tree Task (Raimo et al., 2019), included a frame, on which a star tree topper was depicted, and nine parts of a Christmas tree (left and right lower branches, left and right middle branches, left and right lower branches with trunks, left and right parts of jar, and the top). On a touch screen monitor, the participants were presented with one Christmas tree part at a time, and their task was to place each part on the screen frame where only the topper was depicted as reference part. In particular, a specific part of the tree was shown on the right side of the frame and the participants had to place it at the correct position in the frame with respect to the reference of the star tree topper, by dragging it with their fingers. The position of the part was recorded by the computer in terms of $\mathrm{mm}$ of distance from correct positioning; then, the Christmas tree part disappeared and a new one was presented. Accuracy was evaluated as the sum of $\mathrm{mm}$ of distance from the correct location of each body or tree parts (better performance was indicated by a few $\mathrm{mm}$ ) for both tasks, and the task presentation order was counterbalanced across participants. See Figure 1 for an example of the task items. 


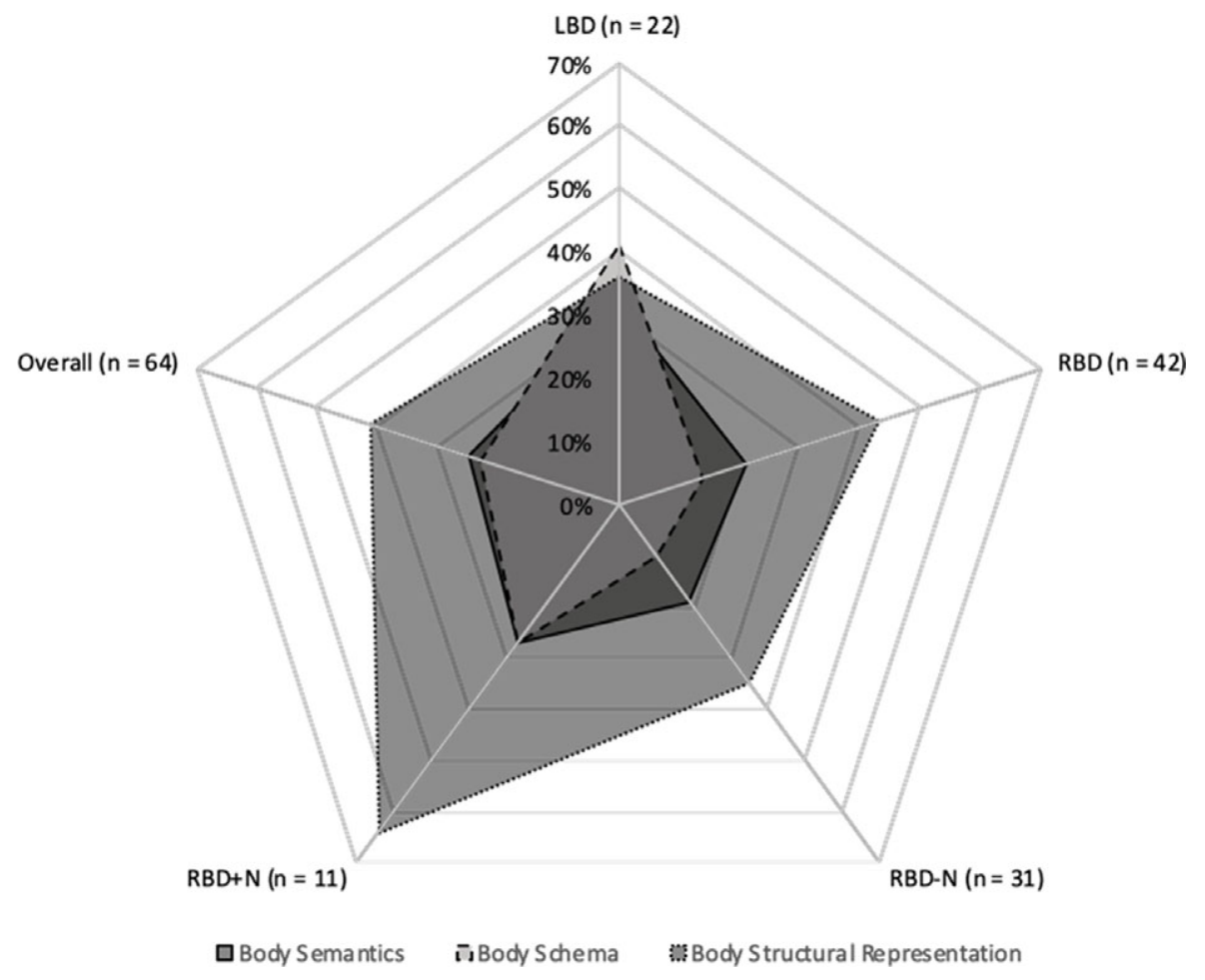

Fig. 2. Side of brain lesion and body representation deficits percentage. LBD, left-brain-damaged patients; RBD, right-brain-damaged patients; RBD-N, right-brain-damaged patients without neglect, RBD+N, right-brain-damaged patients with neglect (i.e., extrapersonal neglect and/or personal neglect).

\section{Assessment of the Body Schema}

Body schema was evaluated using a Hand Laterality Task (adapted and simplified from Parsons, 1987; Schwoebel \& Coslett, 2005; Raimo et al., 2019) in which participants were required to make a decision on the laterality of a single hand (20 stimuli, 10 left and 10 right stimuli) that could be presented at varying degrees of angular rotation $(0,45,90$, 270,315 degrees) on a computer screen. In particular, a rotated hand (target), in the middle of the screen, and a left and a right hand (response items), respectively on the left and right lower part of the touchscreen, were shown. Participants had to decide whether the target hand was the left one or the right one, by mentally rotating the target hand and tapping one of the two response items. Individual scores range from 0 to 20 with higher scores indicating better performance.

The control task included a mental rotation task of nonbody stimuli, the Object Laterality Task (Raimo et al., 2019), where participants were required to make a decision on the laterality of a flower with a leaf positioned at the right or left base of the stem (20 stimuli, 10 left and 10 right stimuli) that could be presented at varying degrees of angular rotation $(0,45,90,270,315$ degrees $)$ on the touch screen. In particular, a rotated flower (target), in the middle of the screen, and a flower with a leaf positioned at the left of the stem and a flower with a leaf positioned at the right of the stem (response items), respectively on the left and right lower part of the touch screen, were shown. Participants had to decide whether the flower target was the one with a leaf positioned at the left or the one with a leaf positioned at the right, by mentally rotating the flower target and tapping one of the two response items. Individual scores range from 0 to 20 with higher scores indicating better performance. To ensure that participants had fully understood the instructions, two practice trials were given for both tasks. Accuracy was recorded for both tasks, and the task presentation order was counterbalanced across participants. See Figure 1 for an example of the task items.

All the tasks were performed on a laptop (13.3" display) equipped with a touch screen monitor, and participants were seated on a chair in front of a desk with the laptop placed upon it. During testing, the participants were instructed to maintain the same position. No time limit was imposed, but they were solicited to respond immediately after presentation.

\section{RESULTS}

\section{Single-Case Analyses}

Crawford's analyses, which were performed by using the computer program SINGLIMS_ES.exe (Crawford \& Howell, 1998; Crawford \& Garthwaite, 2002), to determine whether the scores of individual patients were abnormal and significantly lower than those of the healthy control participants in the BR tasks without considering the performance on control tasks, are extensively reported in Supplementary 
Table 2. Percentage of patients with "selective" deficits in body representation tasks

\begin{tabular}{lccc}
\hline \hline & $\begin{array}{c}\text { "Selective" deficit of } \\
\text { Body Semantics }\end{array}$ & $\begin{array}{c}\text { "Selective" deficit of Body } \\
\text { Structural Representation }\end{array}$ & $\begin{array}{c}\text { "Selective" deficit of } \\
\text { Body Schema }\end{array}$ \\
\hline LBD $(\boldsymbol{n}=\mathbf{2 2})$ & $22.7 \%$ & $18.1 \%$ & $27.2 \%$ \\
RBD $(\boldsymbol{n}=\mathbf{4 2})$ & $11.9 \%$ & $14.2 \%$ & $7.1 \%$ \\
RBD-N $(n=31)$ & $12.9 \%$ & $16.1 \%$ & $3.2 \%$ \\
RBD $+\mathrm{N}(n=11)$ & $9 \%$ & $9 \%$ & $18.1 \%$ \\
Overall $(\mathbf{n}=\mathbf{6 4})$ & $15.6 \%$ & $15.6 \%$ & $14 \%$ \\
\hline \hline
\end{tabular}

LBD, left-brain-damaged patients; RBD, right-brain-damaged patients; RBD-N, right-brain-damaged patients without neglect, $\mathrm{RBD}+\mathrm{N}$, rightbrain-damaged patients with neglect (i.e., extrapersonal neglect and/or personal neglect).

material 3. Overall, these analyses revealed that deficits in at least one BR task were found in $64 \%$ of the patients $(n=41)$. See Figure 2.

To identify patients with a selective deficit of BR (i.e., patients with a worse performance on BR tasks as compared with the respective control tasks) distinguishing between a pure deficit (i.e., a selective deficit in only one BR that is "pure" deficit of body semantics, "pure" deficit of body structural representation, and "pure" deficit of body schema) or mixed deficits of BR (i.e., a selective deficit in two or three body representations), we performed Crawford's analysis using the computer program DISSOCS_ES.exe (Crawford, Garthwaite, \& Porter, 2010) that allows to detect significant dissociation in single cases, by comparing a single case's difference in performance on a BR task and its respective control task with differences observed in the control group.

In eight patients, four LBD [Pt7: $t(1,40)=12.32$, $p<.0001$; Pt16: $t(1,40)=1.78, p=.04$; Pt18: $t(1,40)=$ 1.78, $p=.04$; Pt19: $t(1,40)=1.78, p=.04$ ] and four RBD-N [Pt41: $t(1,40)=10.59, p<.0001 ;$ Pt42: $t(1,40)=$ $1.78, p=.04$; Pt43: $t(1,40)=1.78, p=.04 ; \mathrm{Pt} 48: t(1,40)=$ $1.78, p=.04]$, the differences in performances between the Object-Body Part Association Task and the ObjectRoom Association Task were significantly different from the distribution of differences in controls, demonstrating the presence of a selective and pure impairment in body semantics.

In six patients, two LBD [Pt3: $t(1,40)=3.09, p=.001$; Pt6: $t(1,40)=7.38, p<.0001]$ and four RBD-N [Pt24: $t(1,40)=3.62, p=.0004 ;$ Pt33: $t(1,40)=2.97, p=.002$; Pt36: $t(1,40)=3.37, p=.008 ;$ Pt38: $t(1,40)=1.86, p=.03$ ], the differences in performances between the FBE and the Christmas Tree Task were significantly different from the distribution of differences in controls, demonstrating the presence of a selective impairment in body structural representation.

In six patients, five LBD [Pt4: $t(1,40)=2.10, p=.02 ; \mathrm{Pt} 9$ : $t(1,40)=2.73, p=.004 ;$ Pt10: $t(1,40)=2.42, p=.01 ;$ Pt13: $t(1,40)=3.04, p=.002$; Pt14: $t(1,40)=1.88, p=.03 ;]$ and one RBD-N [Pt51: $t(1,40)=1.98, p=.02]$, the differences in performances between the Hand Laterality Task and the Object Laterality Task were significantly different from the distribution of differences in controls, demonstrating the presence of a selective and pure impairment in body schema.
In three patients, one LBD [Pt5; association tasks: $t(1,40)=7.10, p<.0001$; structural representation tasks: $t(1,40)=11.76, p<.001]$, one RBD-N [Pt39; association tasks: $t(1,40)=5.33, p<.0001$; structural representation tasks: $t(1,40)=4.15, p<.001]$, and one RBD+N [Pt64; association tasks: $t(1,40)=5.33, p<.0001$; structural representation tasks: $t(1,40)=4.15, p<.001]$, the differences in performances between the Object-Body Part Association Task and the Object-Room Association Task, and the differences between FBE and the Christmas Tree Task were significantly different from the distributions of differences in controls, demonstrating the presence of a mixed selective impairments in body semantics and body structural representation.

In two patients, one LBD [Pt11; structural representation tasks: $t(1,40)=2.17, p=.01$; laterality tasks: $t(1,40)=2.10$, $p=.02 ;]$ and one $\mathrm{RBD}+\mathrm{N}$ [Pt63; structural representation tasks: $t(1,40)=6.04, p<.0001$; laterality tasks: $t(1,40)=$ 2.73, $p=.004]$, the differences in performances between the FBE and the Christmas Tree Task and the differences between the Hand Laterality Task and the Object Laterality Task were significantly different from the distributions of differences in controls, demonstrating the presence of mixed selective impairments in body structural representation and body schema. See Supplementary material 3 for details on individual patients' scores on BR and control tasks and on single-case analyses. Further details on the percentages of patients with a "selective" and "pure" BR deficit considering the patient groups (LBD, RBD-N, RBD+N) are reported in Tables 2 and 3 and showed in Figures 3 and 4.

\section{Group Analyses}

Group analyses were performed to evaluate the overall effect of unilateral brain damage on BRs and a possible hemispheric lateralization of BRs, disentangling the assessment of BRs from other cognitive skills required to perform the BR tasks. Kolmogorov-Smirnov test was applied to verify the normal distribution of data on accuracy scores; since the results showed that data were not normally distributed, nonparametric analyses were performed. In particular to disentangle the contribution of other cognitive skills involved in performing BR tasks and investigating the significant difference on BR tasks among groups, three Quade's rank analyses of 
Table 3. Percentage of patients with a "pure" deficit in one of the three body representations

\begin{tabular}{lccc}
\hline \hline & $\begin{array}{c}\text { "Pure" deficit of Body } \\
\text { Semantics }\end{array}$ & $\begin{array}{c}\text { "Pure” Deficit of Body Structural } \\
\text { Representation }\end{array}$ & $\begin{array}{c}\text { "Pure" Deficit of Body } \\
\text { Schema }\end{array}$ \\
\hline LBD $(\boldsymbol{n}=\mathbf{2 2})$ & $18.1 \%$ & $9 \%$ & $22.7 \%$ \\
RBD $(\boldsymbol{n}=\mathbf{4 2})$ & $9.5 \%$ & $9.5 \%$ & $2.3 \%$ \\
RBD-N $(n=31)$ & $12.9 \%$ & $12.9 \%$ & $3.2 \%$ \\
RBD $+\mathrm{N}(n=11)$ & $0 \%$ & $0 \%$ & $0 \%$ \\
Overall $(\boldsymbol{n}=\mathbf{6 4})$ & $12.5 \%$ & $9.3 \%$ & $9.3 \%$ \\
\hline \hline
\end{tabular}

LBD, left-brain-damaged patients; RBD, right-brain-damaged patients; RBD-N, right-brain-damaged patients without neglect, $\mathrm{RBD}+\mathrm{N}$, rightbrain-damaged patients with neglect (i.e., extrapersonal neglect and/or personal neglect).

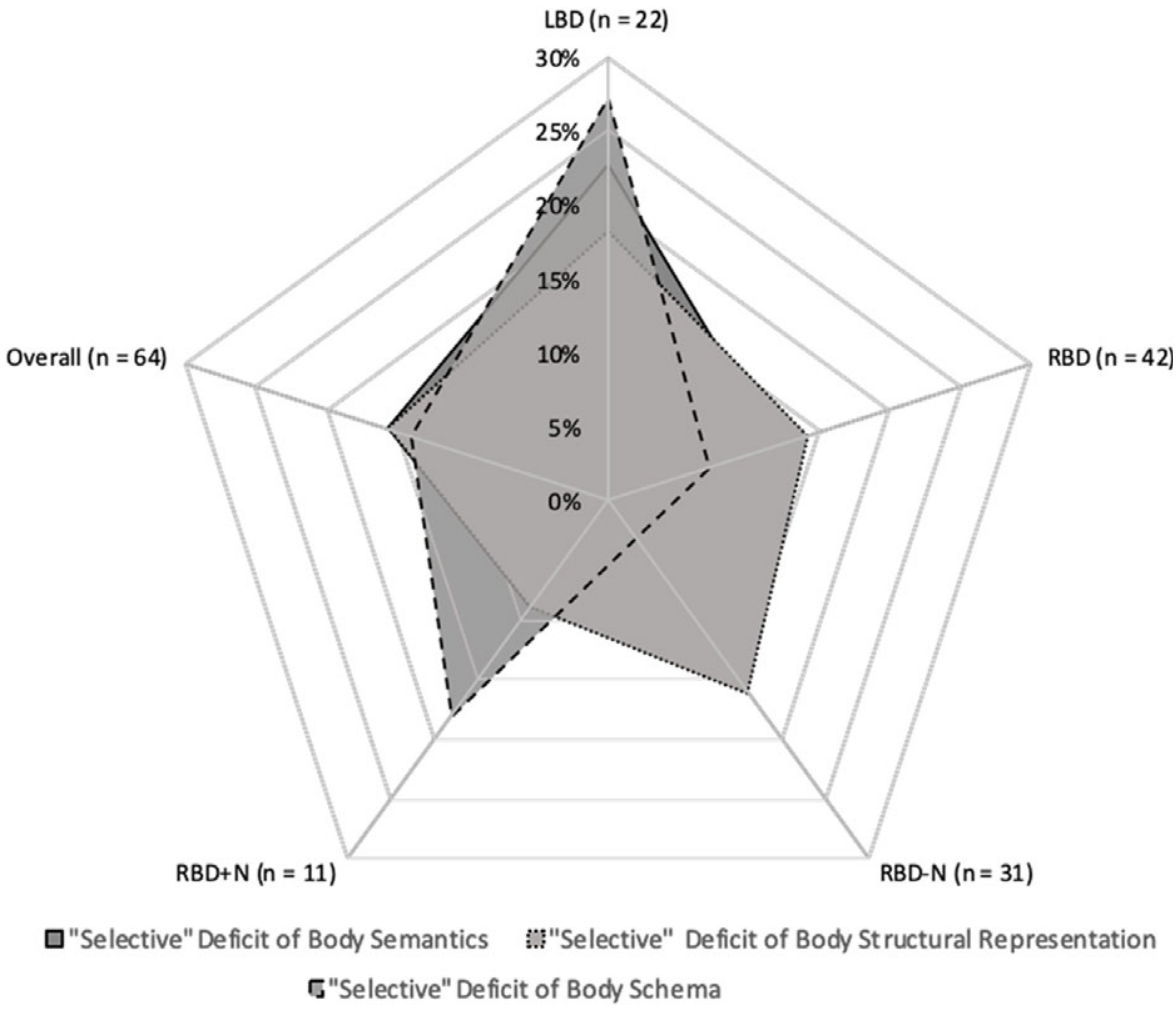

Fig. 3. Side of brain lesion and "selective" body representation deficits percentage. LBD, left-brain-damaged patients; RBD, right-braindamaged patients; RBD-N, right-brain-damaged patients without neglect, RBD+N, right-brain-damaged patients with neglect (i.e., extrapersonal neglect and/or personal neglect).

covariance were performed, following these steps: (i) we ranked the scores of each $\mathrm{BR}$ task (dependent variables: Object-Body Part Association Task; FBE; Hand Laterality Task) and of each control task (covariates: Object-Room Association Task; Christmas Tree Task; Object Laterality Task); (ii) we performed three linear regression analyses of the ranks of the dependent variable on the ranks of the covariate, saving the residuals. In particular, the first analysis performed a regression of the ranks of Object-Body Part Association Task on the ranks of the Object-Room Association Task (i.e., the respective control task), and body semantic residuals were calculated; the second analysis performed a regression of the ranks of the FBE on the ranks of the Christmas Tree Task (i.e., the respective control task), and body structural representation residuals were calculated; and the third regression analysis performed a regression of the ranks of the Hand Laterality Task on the ranks of the Object Laterality Task, and body schema residuals were calculated; (iii) to implement the Quade's test computed by using IBM SPSS Statistics for Macintosh, Version 24.0, we performed three analyses of variance (ANOVA) with body semantics, or body structural representation, or body schema residuals as dependent variable, and the Group (LBD, RBD-N, $\mathrm{RBD}+\mathrm{N}, \mathrm{C}$ ) as independent variable and years of education as covariate. The $\mathrm{F}$ test resulting from this ANOVA was the $F$ statistic Quade's test. Moreover, to analyse significant effects, Mann-Whitney $U$ was performed and a Bonferroni correction for multiple comparisons was applied.

Concerning the body semantics (Object-Body Part Association Task), the rank analysis of covariance 


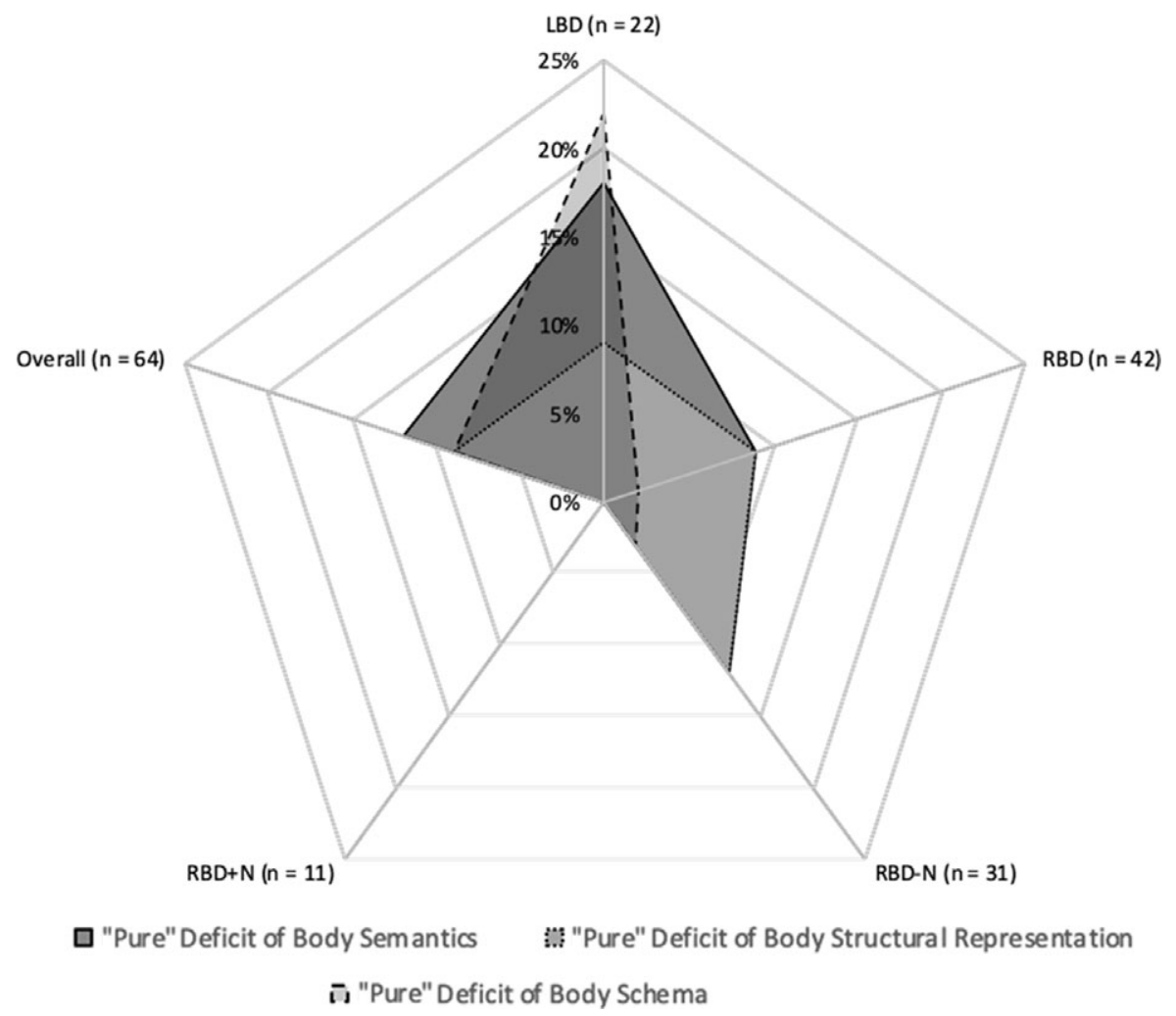

Fig. 4. Side of brain lesion and "pure" body representation deficits percentage. LBD, left-brain-damaged patients; RBD, right-brain-damaged patients; RBD-N, right-brain-damaged patients without neglect, RBD+N, right-brain-damaged patients with neglect (i.e., extrapersonal neglect and/or personal neglect).

(Quade's test) showed no significant main effect of Group $\left(F_{(3,103)}=1.57 ; p=.201\right)$ even when the years of education were added as covariate $\left(F_{(3,103)}=1.43 ; p=.239\right)$.

Concerning the body structural representation (FBE Task), the rank analysis of covariance showed a significant effect of Group $\left(F_{(3,103)}=5.18 ; p=.002\right)$ even when the years of education were added as covariate $\left(F_{(3,103)}=\right.$ $5.14 ; p=.002)$. Post-hoc comparison showed that all patient groups performed significantly worse than controls (LBD vs. C: Mann-Whitney $U=187, p<.0001$; RBD-N vs. C Mann-Whitney $U=283, p<.0001$; RBD $+\mathrm{N}$ vs. C: Mann-Whitney $U=45, p<.0001$ ) while the three patient groups showed a similar performance (LBD vs. RBD-N: Mann-Whitney $U=336, p=.928$; LBD vs. RBD+N: Mann-Whitney $U=77, p=.093$; RBD-N vs. RBD+N: Mann-Whitney $U=111, p=.089)$.

Concerning the body schema (Hand Laterality Task), the rank analysis of covariance (Quade's test) showed a significant effect of the Group $\left(F_{(3,103)}=4.07, p=.009\right)$ even when the years of education were added as covariate $\left(F_{(3,103)}=3.43, p=.019\right)$. Post-hoc comparison showed that the LBD performed significantly worse than both $\mathrm{C}$ (Mann-Whitney $U=241, p=.002$ ) and RBD-N (MannWhitney $U=228, p=.037$ ), but no significant differences were found between LBD and RBD+N (Mann-Whitney $U=84, p=.166$ ). Both the RBD groups performed similarly to controls (RBD-N vs. C: Mann-Whitney $U=528$, $p=.196 ; \mathrm{RBD}+\mathrm{N} v s$. C: Mann-Whitney $U=176, p=.237)$ and to each other (RBD-N vs. RBD+N: Mann-Whitney $U=160, p=.767)$.

All the significant differences survived after Bonferroni correction for multiple comparisons, but the difference between LBD and RBD-N on the Hand Laterality Task $(p=.037)$. Means and standard deviations of accuracy of performance on the BR for each group are shown in Table 4.

\section{DISCUSSION}

The aim of the present study was to evaluate the three different BRs (the body schema, the body structural representation, and the body semantics) within the same sample of braindamaged patients, disentangling the contribution of other cognitive skills involved in performing BR tasks and investigating the specific impact of left or right unilateral brain damage.

The incidence of disorders of BRs was very high in our sample when we considered the performance only in BR tasks (see Supplementary material 3 for details). Indeed, $64 \%$ of patients with unilateral brain damage were impaired, as compared with controls, on at least one BR task. This result is in line with the rate reported in the seminal study by Schwoebel and Coslett (2005), showing that $51 \%$ of patients with unilateral stroke were impaired on at least one BR 
Table 4. Means and standard deviations on the body representation tasks in the four groups

\begin{tabular}{|c|c|c|c|c|}
\hline & $\mathrm{C}$ & LBD & RBD-N & $\mathrm{RBD}+\mathrm{N}$ \\
\hline \multicolumn{5}{|l|}{ Body Semantics } \\
\hline Object-Body Part Association Task (range 0-20) & $19.88 \pm .41$ & $18.81 \pm 2.46$ & $19.42 \pm 1.65$ & $19.82 \pm .40$ \\
\hline Object-Room Association Task (range 0-20) & $19.88 \pm .42$ & 18.18. \pm 1.59 & $19.56 \pm .79$ & $19.10 \pm 1.10$ \\
\hline \multicolumn{5}{|l|}{ Body Structural Representation } \\
\hline Frontal Body Evocation Task (distance in $\mathrm{mm}$ ) & $75.83 \pm 27.83$ & $136.40 \pm 110.14$ & $117.28 \pm 48.80$ & $159.88 \pm 72.72$ \\
\hline Christmas Tree Task (distance in $\mathrm{mm}$ ) & $140.18 \pm 73.14$ & $187.09 \pm 95.69$ & $182.66 \pm 72.97$ & $265.58 \pm 119.01$ \\
\hline \multicolumn{5}{|l|}{ Body Schema } \\
\hline Hand Laterality Task (range 0-20) & $18.46 \pm 2.54$ & $15.50 \pm 4.13$ & $17.71 \pm 3.06$ & $17.73 \pm 3.23$ \\
\hline Object Laterality Task (range 0-20) & $18.66 \pm 3.68$ & $18.27 \pm 3.01$ & $16.97 \pm 2.99$ & $17.51 \pm 3.17$ \\
\hline
\end{tabular}

C, control participants; LBD, left-brain-damaged patients; RBD-N, right-brain-damaged patients without neglect, RBD+N, right-brain-damaged patients with neglect (i.e., extrapersonal neglect and/or personal neglect).

measure. Thus current results, derived from a comparison with a very robust single case methodology and using a larger sample of healthy controls including both men and women (i.e., $n=41$ vs. 18 healthy controls who were all women but one in Schowoebel \& Coslett, 2005), further suggest that the body processing is often compromised after brain damage. Instead, Razmus (2017) showed that $81 \%$ of patients were impaired on at least one BR measure. However, the higher incidence of BR deficits in the study by Razmus (2017) could be related to the bilateral lesions that occurred in most cases while the present study and the one by Schwoebel's and Coslett's assessed only patients with unilateral brain damage.

An innovative aspect of the current study is the possibility to detect selective BR since, differently from these relevant previous studies (Schwoebel \& Coslett, 2005; Razmus, 2017), we controlled for the possible effect of cognitive skills required to perform the BR tasks (e.g., in visual processing, mental imagery, visuospatial attention, decision-making, etc.). The incidence of BR disorders was lower in our sample when performance on control tasks was considered: $37.5 \%$ of patients with unilateral brain damage were, indeed, impaired on at least one BR task, relative to controls, when compared with the respective control tasks (selective deficits). Moreover, we also identified pure deficits (i.e., a deficit in only one specific BR) in patients with unilateral brain damage finding that these were present in $31.2 \%$ of patients: $12.5 \%$ showed a selective deficit of the body semantics; $9.37 \%$ a selective deficit of the body structural representation; and $9.37 \%$ a selective deficit of the body schema. With respect to the hemispheric laterality, a selective and pure deficit in body schema was frequently detected in the sample of left-brain-damaged patients (i.e., selective deficit: $27.2 \%$ of the patients with LBD vs. $7.1 \%$ of the patients with RBD; pure deficit: $22.7 \%$ of the patients with LBD vs. $2.3 \%$ of the patients with RBD).

The presence of pure BR deficits is noteworthy since it underlines a certain degree of independence between these three BRs, at least in adulthood. In other words, although these three BRs can shape each other, above all during development (see Raimo et al., 2019; Aucliar \& Jambaqué, 2015), and there is evidence that their construction is based on their interactions (Pitron et al., 2018), the selective pure deficits reported in this study further prove that they are functionally distinct.

Furthermore, we performed specific nonparametric statistical analyses (Quade's rank analysis of covariance) that allowed us to evaluate a possible hemispheric lateralization and the overall effect of unilateral brain damage on BR processing, regardless other (and more general) cognitive processes required to perform BR tasks. In this case, the three patient groups (LBD, RBD-N, RBD $+\mathrm{N}$ ) performed similarly to controls on the body semantics task (i.e., the Object-Body Part Association Task), whereas their performances were significantly worse than those of controls on the body structural representation (i.e., the FBE Task). Moreover, the LBD group showed performances significantly worse than those of controls and RBD-N on the body schema (i.e., Hand Laterality Task), although this latter comparison did not survive multiple comparison testing.

Current findings showed that the left hemisphere could play a pivotal role in the body schema in consistence with the previous neuropsychological literature finding that deficits in BR generally follow left hemispheric lesions (see, e.g., Semenza, 2001). In particular, previous functional studies (Tomasino, Toraldo, \& Rumiati, 2003; Kosslyn, DiGirolamo, Thompson, \& Alpert, 1998) assessing mental rotation of body (hand) and no-body (i.e., flag shapes, 3D cubes) stimuli showed that hand laterality judgments engendered activation in the left hemisphere, while laterality judgments of no body objects engendered activation in the right hemisphere, suggesting that only patients with left hemisphere lesions would be selectively impaired in mental rotation of body stimuli requiring motor strategy and body schema activation (Tomasino \& Rumiati, 2004). However, this result should be cautiously interpreted because the difference between LBD and RBD-N on Hand Laterality Task performances did not survive correction for multiple comparisons.

Current results suggest that the right and left hemispheres are both involved in the processing of the body structural 
representation, since this representation is specifically compromised in both left- and right-brain-damaged patients. The correct location of each body part has been suggested to involve the posterior parietal cortex and the intraparietal sulcus in the left side of the brain (Denes, Cappelletti, Zilli, Dalla Porta, \& Gallana, 2000; Schwoebel, Coslett, \& Buxbaum, 2001; Felician, Ceccaldi, Didic, Thinus-Blanc, \& Poncet, 2003), so that damage to these regions may lead to autotopagnosia (Felician et al., 2004). However, the body structural representation largely derived from visual input of others' bodies through a self/other processing that has been suggested to have its neural underpinnings in frontoparietal cortex of the right side of the brain (Decety \& Sommerville, 2003; Vogeley \& Fink, 2003; Blanke \& Arzy, 2005). The role of the right hemisphere in processing spatial knowledge about the body is also strongly supported by previous studies on unilateral brain-damaged patients (Di Vita et al., 2017; Di Vita, Palermo, Boccia, \& Guariglia, 2019; Palermo et al., 2014) showing that deficit in tasks tapping on the body structural representation usually results from lesions within the right hemisphere, so much so that body structural representation deficit may be considered a clinical sign of personal neglect (Di Vita et al., 2017, 2019). Our finding on the body structural representation allows to reconciliate previous data on stroke patients that suggested a prominent role of only one of the brain hemispheres (i.e., the right hemisphere in Di Vita et al., 2017; 2019; Palermo et al., 2014; and the left hemisphere in Schwoebel \& Coslett, 2005) and to better discuss some issues that remained unresolved in previous literature, due to small sample sizes (e.g., Di Vita et al., 2017) or to failure to assess this BR taking into account possible deficits in other cognitive processes necessary to perform the task (e.g., Di Vita et al., 2019; Palermo et al., 2014; Schwoebel \& Coslett, 2005).

In sum, our study confirms the hypothesis that BR deficits are a common consequence of left and right unilateral brain damage, excluding any doubt about the fact that these deficits are not due to more general impairments in cognitive functioning.

Considering the BR theoretical framework on the basis of the triadic taxonomy (Schwoebel \& Coslett, 2005; Sirigu et al., 1991), our results show that selective and pure deficits in the conceptual and linguistic representation of the body (i.e., body semantics), in the sensory-motor representation of the body (i.e., body schema), and in the visuospatial map of the different body parts (i.e., body structural representation) were detected with the same frequency in this population of patients with brain damage. Furthermore, while a deficit of the body structural representation is a common consequence of lesions to both hemispheres, a deficit of the body schema would seem to be associated to the left brain damage. Therefore, current results shed precious light on how specific BR deficits, such as other cognitive dysfunctions (i.e., aphasia, apraxia, memory deficits), may be a consequence of unilateral brain damage and are not secondary to other more general cognitive deficits.
We used a battery of tasks specifically developed to assess the three BRs patterned upon the tasks used by Schwoebel and Coslett (2005) and in line with the previous literature (see Razmus, 2017; Di Vita et al., 2019). Possible caveats are due to the use of only one task to assess each BR and to differences between the tasks developed to test different $\mathrm{BR}$ and relative scoring procedures (e.g., body schema and body semantics were assessed by means of choosing between two alternatives, whereas body structural representation was assessed by means of correct location of a stimulus in a frame measured in $\mathrm{mm}$ of distance). Therefore, further normative studies should be conducted to establish the different sensitivity of these tasks to assess each BR specifically. Moreover, we emphasize the need to evaluate BRs in clinical practice since they may be altered in various ways after central nervous system damage and result in a vast range of clinical manifestations (see de Vignemont, 2010). Further, BR deficits are often underreported due to the lack of a detailed exploration of a patient's BR in routine neurological assessment (Antoniello et al., 2010). BR deficits may affect motor outcomes, daily functioning, and quality of life of patients with unilateral brain damage, suggesting that these patients could benefit from specific neuropsychological rehabilitation training targets to treat BR disorders in association with standard rehabilitation training programs.

\section{ACKNOWLEDGMENTS}

We are thankful to Stefano Buratin and Francesco Ruggeri for their help with the development of the computerized version of the experimental tasks, to Stefano Buratin for his help with the development of the computerized version of the scoring sheets, to Professor Maurizio Iocco for allowing access to some patients, and to Maria Giovanna Caruso for helping with the recruitment of some patients.

\section{FUNDING}

This research has been supported by funding from Fondazione con il Sud to Liana Palermo (Brains2South Project code: 2015-PDR-0248).

\section{CONFLICT OF INTEREST}

The authors have nothing to disclose.

\section{SUPPLEMENTARY MATERIAL}

To view supplementary material for this article, please visit https://doi.org/10.1017/S1355617721000151

\section{REFERENCES}

Antoniello, D., Kluger, B.M., Sahlein, D.H., \& Heilman, K.M. (2010). Phantom limb after stroke: An underreported 
phenomenon. Cortex, 46(9), 1114-1122. doi: 10.1016/j.cortex. 2009.10.003

Auclair, L. \& Jambaqué, I. (2015). Lexical-semantic body knowledge in 5- to 11-year-old children: How spatial body representation influences body semantics. Child Neuropsychology, 21(4), 451-464. doi: 10.1080/09297049.2014.912623

Berlucchi, G. \& Aglioti, S.. (2010). The body in the brain revisited. Experimental Brain Research, 200(1), 25-35. doi: 10.1007/s00 221-009-1970-7

Blanke, O. \& Arzy, S. (2005). The out-of-body experience: Disturbed self-processing at the temporo-parietal junction. The Neuroscientist, 11(1), 16-24. doi: 10.1177/107385840427 0885

Blanke, O. \& Mohr, C. (2005). Out-of-body experience, heautoscopy, and autoscopic hallucination of neurological origin Implications for neurocognitive mechanisms of corporeal awareness and self-consciousness. Brain research. Brain research reviews, 50(1), 184-199. doi: 10.1016/j.brainresrev. 2005.05.008

Buxbaum, L.J. \& Branch Coslett, H. (2001). Specialised structural descriptions for human body parts: Evidence from autotopagnosia. Cognitive Neuropsychology, 18(4), 289-306. doi: 10.1080/02643290126172

Buxbaum, L.J., Giovannetti, T., \& Libon, D. (2000). The role of the dynamic body schema in praxis: Evidence from primary progressive apraxia. Brain and cognition, 44(2), 166-191. doi: 10.1006/brcg.2000.1227

Canzano, L., Piccardi, L., Bureca, I., \& Guariglia, C. (2011). Mirror writing resulting from an egocentric representation disorder: A case report. Neurocase, 17(5), 447-460. doi: 10.1080/13554 794.2010.532143

Committeri, G., Piervincenzi, C., \& Pizzamiglio, L. (2018). Personal neglect: A comprehensive theoretical and anatomoclinical review. Neuropsychology, 32(3), 269-279. doi: 10.1037/ neu0000409

Committeri, G., Pitzalis, S., Galati, G., Patria, F., Pelle, G., Sabatini, U., Castriota-Scanderbeg, A., Piccardi, L., Guariglia, C., \& Pizzamiglio, L. (2007). Neural bases of personal and extrapersonal neglect in humans. Brain, 130, 431-441. doi: 10.1093/ brain/awl265

Corradi-Dell'Acqua, C. \& Rumiati, R.I. (2007). What the brain knows about the body: Evidence for dissociable representations. Brain Development in Learning Environments: Embodied and Perceptual Advancements. Newcastle: Cambridge Scholar Publishing, 50-64.

Coslett, H.B., Saffran, E.M., \& Schwoebel, J. (2002). Knowledge of the human body: A distinct semantic domain. Neurology, 59(3), 357-363. doi: 10.1212/wnl.59.3.357

Crawford, J.R. \& Garthwaite, P.H. (2002). Investigation of the single case in neuropsychology: Confidence limits on the abnormality of test scores and test score differences. Neuropsychologia, 40(8), 1196-1208. doi: 10.1016/s0028-3932 (01)00224-x

Crawford, J.R., Garthwaite, P.H., \& Porter, S. (2010). Point and interval estimates of effect sizes for the case-controls design in neuropsychology: Rationale, methods, implementations, and proposed reporting standards. Cognitive Neuropsychology, 27, 245-260. doi: 10.1080/02643294.2010.513967

Crawford, J.R. \& Howell, D.C. (1998). Comparing an individual's test score against norms derived from small samples. Clinical Neuropsychologist, 12(4), 482-486. doi: 10.1076/clin.12.4.482. 7241
Daurat-Hmeljiak, C., Stambak, M., \& Berges, J. (1978). Il test dello schema corporeo. Una prova di conoscenza e costruzione dell'immagine del corpo [The body schema test. A test of knowledge and construction of body image]. In Firenze. Organizzazioni Speciali.

de Vignemont F. (2010). Body schema and body image - pros and cons. Neuropsychologia, 48(3), 669-680. doi: 10.1016/j.neuro psychologia.2009.09.022

de Vignemont, F. (2011). Embodiment, ownership and disownership. Consciousness and cognition, 20(1), 82-93. doi: 10.1016/ j.concog.2010.09.004

Decety, J. \& Sommerville, J.A. (2003). Shared representations between self and other: A social cognitive neuroscience view. Trends in cognitive sciences, 7(12), 527-533. doi: 10.1016/j. tics.2003.10.004

Denes, G., Cappelletti, J.Y., Zilli, T., Dalla Porta, F., \& Gallana, A. (2000). A category-specific deficit of spatial representation: The case of autotopagnosia. Neuropsychologia, 38(4), 345-350. doi: 10.1016/s0028-3932(99)00101-3

Di Vita, A., Cinelli, M.C., Raimo, S., Boccia, M., Buratin, S., Gentili, P., Inzitari, M.T., Iona, T., Iosa, M., Morelli, D., Ruggeri, F., Russo, G., Guariglia, C., \& Palermo, L. (2020). Body representations in children with cerebral palsy. Brain sciences, 10(8), E490. doi: 10.3390/brainsci10080490

Di Vita, A., Palermo, L., Boccia, M., \& Guariglia, C. (2019). Topological map of the body in post-stroke patients: Lesional and hodological aspects. Neuropsychology, 33(4), 499. doi: 10. 1037/neu0000536

Di Vita, A., Palermo, L., Piccardi, L., Di Tella, J., Propato, F., \& Guariglia, C. (2017). Body representation alterations in personal but not in extrapersonal neglect patients. Applied Neuropsychology: Adult, 24(4), 308-317. doi: 10.1080/23279095.2016.1174866

Felician, O., Ceccaldi, M., Didic, M., Thinus-Blanc, C., \& Poncet, M. (2003). Pointing to body parts: A double dissociation study. Neuropsychologia, 41(10), 1307-1316. doi: 10.1016/s0028-39 32(03)00046-0

Felician, O., Romaiguère, P., Anton, J.L., Nazarian, B., Roth, M., Poncet, M., \& Roll, J.P. (2004). The role of human left superior parietal lobule in body part localization. Annals of Neurology: Official Journal of the American Neurological Association and the Child Neurology Society, 55(5), 749-751. doi: 10.1002/ana. 20109

Fontes, P.L., Cruz, T.K.F., Souto, D.O., Moura, R., \& Haase, V.G. (2017). Body representation in children with hemiplegic cerebral palsy. Child Neuropsychol., 23(7), 838-863. http://dx.doi.org:10. 1080/09297049.2016.1191629

Fontes, P.L., Moura, R., \& Haase, V.G. (2014). Evaluation of body representation in children with hemiplegic cerebral palsy: Toward the development of a neuropsychological test battery. Psychology \& Neuroscience, 7(2), 139-149. http://dx.doi.org/10.3922/j.psns. 2014.01

Gandola, M., Invernizzi, P., Sedda, A., Ferrè, E.R., Sterzi, R., Sberna, M., Paulesu, E., \& Bottini, G. (2012). An anatomical account of somatoparaphrenia. Cortex, 48(9), 1165-1178. doi: 10.1016/j.cortex.2011.06.012

Guariglia, C. \& Antonucci, G. (1992). Personal and extrapersonal space: A case of neglect dissociation. Neuropsychologia, 30(11), 1001-9. doi: 10.1016/0028-3932(92)90051-m

Guariglia, C., Palermo, L., Piccardi, L., Iaria, G., \& Incoccia, C. (2013). Neglecting the left side of a city square but not the left side of its clock: Prevalence and characteristics of representational neglect. PLoS One, 8(7), e67390. doi: 10.1371/journal.pone. 0067390 
Guariglia, C., Piccardi, L., Allegra, M.P., \& Traballesi, M. (2002). Is autotopoagnosia real? EC says yes. A case study. Neuropsychologia, 40(10), 1744-1749. doi: 10.1016/s00283932(02)00013-1

Herbet, G., Lemaitre, A.L., Moritz-Gasser, S., Cochereau, J., \& Duffau, H. (2019). The antero-dorsal precuneal cortex supports specific aspects of bodily awareness. Brain, 142(8), 2207-2214. doi: 10.1093/brain/awz179

Karnath, H.O. \& Baier, B. (2010). Right insula for our sense of limb ownership and self-awareness of actions. Brain Structure and Function, 214(5-6), 411-417. doi: 10.1007/s00429-0100250-4

Kosslyn, S.M., DiGirolamo, G.J., Thompson, W.L., \& Alpert, N.M. (1998). Mental rotation of objects versus hands: Neural mechanisms revealed by positron emission tomography. Psychophysiology, 35(2), 151-161. doi: 10.1111/1469-8986.3520151

Laiacona, M., Allamano, N., Lorenzi, L., \& Capitani, E. (2006). A case of impaired naming and knowledge of body parts. Are limbs a separate sub-category?. Neurocase, 12(5), 307-316. doi: 10.1080/13554790601125940

Moro, V., Pernigo, S., Tsakiris, M., Avesani, R., Edelstyn, N.M., Jenkinson, P.M., \& Fotopoulou, A. (2016). Motor versus body awareness: Voxel-based lesion analysis in anosognosia for hemiplegia and somatoparaphrenia following right hemisphere stroke. Cortex, 83, 62-77. doi: 10.1016/j.cortex.2016. 07.001

Paillard, J. (1980). Le corps situé et le corps identifié. Revue medicale de la Suisse romande, 100(2), 129-141.

Palermo, L., Di Vita, A., Piccardi, L., Traballesi, M., \& Guariglia, C. (2014). Bottom-up and top-down processes in body representation: A study of brain-damaged and amputee patients. Neuropsychology, 28(5), 772-781. doi: 10.1037/neu0000086

Paolucci, S., Bureca, I., Multari, M., Nocentini, U., \& Matano, A. (2010). An open-label pilot study of the use of rivastigmine to promote functional recovery in patients with unilateral spatial neglect due to first ischemic stroke. Functional Neurology, 25(4), 195-200.

Parsons, L.M. (1987). Imagined spatial transformations of one's hands and feet. Cognitive Psychology, 19(2), 178-241. doi: 10. 1016/0010-0285(87)90011-9

Pitron, V., Alsmith, A., \& de Vignemont, F. (2018). How do the body schema and the body image interact?. Consciousness and Cognition, 65, 352-358. doi: 10.1016/j.concog.2018.08.007

Pizzamiglio, L., Judica, A., Razzano, C., \& Zoccolotti, P. (1989). Toward a comprehensive diagnosis of visual-spatial disorders in unilateral brain damaged patients. Psychological Assessment, 5, 199-218.

Raimo, S., Boccia, M., Di Vita, A., Iona, T., Cropano, M., Ammendolia, A., Colao, R., Iocco, M., Angelillo, V., Guariglia, C., Grossi, D., Palermo, L. (2020). Interoceptive awareness in focal brain-damaged patients. Neurological Sciences, 41(6), 1627-1631. doi: 10.1007/s10072-019-04172-z

Raimo, S., Boccia, M., Di Vita, A., Cropano, M., Guariglia, C., Grossi, D., \& Palermo, L. (2021). The Body across adulthood: on the relation between interoception and body representations. Frontiers in Neuroscience, 15, 586684. doi: 10.3389/fnins. 2021.586684
Raimo, S., Iona, T., Di Vita, A., Boccia, M., Buratin, S., Ruggeri, F., Iosa, M., Guariglia, C., Grossi, D., \& Palermo, L. (2019). The development of body representations in school-aged children. Applied Neuropsychology: Child, 1-13. doi: 10.1080/ 21622965.2019.1703704

Raven, J.C. (1938). Standard Progressive Matrices: Sets A, B, C, D and $E H K$. London: Lewis.

Razmus, M. (2017). Body representation in patients after vascular brain injuries. Cognitive processing, 18(4), 359-373. doi: 10. 1007/s10339-017-0831-8

Schwoebel, J., Boronat, C.B., \& Coslett, H.B. (2002). The man who executed "imagined" movements: Evidence for dissociable components of the body schema. Brain and Cognition, 50(1), 1-16. doi: 10.1016/s0278-2626(02)00005-2

Schwoebel, J. \& Coslett, H.B. (2005). Evidence for multiple, distinct representations of the human body. Journal of Cognitive Neuroscience, 17(4), 543-553. doi: 10.1162/ 0898929053467587

Schwoebel, J., Coslett, H.B., \& Buxbaum, L.J. (2001). Compensatory coding of body part location in autotopagnosia: Evidence for extrinsic egocentric coding. Cognitive Neuropsychology, 18(4), 363-381. doi: 10.1080/02643290126218

Semenza, C. (2001). Disorder of body representation. In R.S. Berndt (Ed.), Handbook of Neuropsychology (Vol. 3, pp. 285-303). Amsterdam, The Netherlands: Elsevier Science BV.

Sirigu, A., Grafman, J., Bressler, K., \& Sunderland, T. (1991). Multiple representations contribute to body knowledge processing: Evidence from a case of autotopagnosia. Brain, 114(1), 629-642. doi: 10.1093/brain/114.1.629

Spinnler, H. \& Tognoni, G. (1987). Standardizzazione e taratura italiana di tests neuropsicologici. [Italian normative values and standardization of neuropsychological tests]. Italian Journal of Neurological Sciences, 6(Suppl. 8), 1-20. doi: 10.1007/BF023 37583

Suzuki, K., Yamadori, A., \& Fuji, T. (1997). Category-specific comprehension deficit restricted to body parts. Neurocase, 3(3), 193-200. doi: 10.1080/13554799708404054

Tomasino, B. \& Rumiati, R.I. (2004). Effects of strategies on mental rotation and hemispheric lateralization: Neuropsychological evidence. Journal of cognitive neuroscience, 16(5), 878-888. doi: 10.1162/089892904970753

Tomasino, B., Toraldo, A., \& Rumiati, R.I. (2003). Dissociation between the mental rotation of visual images and motor images in unilateral brain-damaged patients. Brain and Cognition, 51(3), 368-371. doi: 10.1016/s0278-2626(02)00570-5

Vallar, G. \& Ronchi, R. (2009). Somatoparaphrenia: A body delusion. A review of the neuropsychological literature. Experimental brain research, 192(3), 533-551. doi: 10.1007/ s00221-008-1562-y

Vogeley, K. \& Fink, G.R. (2003). Neural correlates of the firstperson-perspective. Trends in cognitive sciences, 7(1), 38-42. doi: 10.1016/s1364-6613(02)00003-7

Zoccolotti, P., Antonucci, G., \& Judica, A. (1992). Psychometric characteristics of two semi-structured scales for the functional evaluation of hemi-inattention in extrapersonal and personal space. Neuropsychological Rehabilitation, 2(3), 179-191. https://psycnet.apa.org/doi/10.1080/09602019208401407 